\title{
Threat of Punishment Motivates Memory Encoding via Amygdala, Not Midbrain, Interactions with the Medial Temporal Lobe
}

\author{
Vishnu P. Murty, ${ }^{1,2}$ Kevin S. LaBar, ${ }^{1,3,4}$ and R. Alison Adcock ${ }^{1,2,3,4}$ \\ ${ }^{1}$ Center for Cognitive Neuroscience and Departments of ${ }^{2}$ Neurobiology, ${ }^{3}$ Psychology and Neuroscience, and ${ }^{4}$ Psychiatry and Behavioral Sciences, Duke \\ University, Durham, North Carolina 27708
}

\begin{abstract}
Neural circuits associated with motivated declarative encoding and active threat avoidance have both been described, but the relative contribution of these systems to punishment-motivated encoding remains unknown. The current study used functional magnetic resonance imaging in humans to examine mechanisms of declarative memory enhancement when subjects were motivated to avoid punishments that were contingent on forgetting. A motivational cue on each trial informed participants whether they would be punished or not for forgetting an upcoming scene image. Items associated with the threat of shock were better recognized $24 \mathrm{~h}$ later. Punishmentmotivated enhancements in subsequent memory were associated with anticipatory activation of right amygdala and increases in its functional connectivity with parahippocampal and orbitofrontal cortices. On a trial-by-trial basis, right amygdala activation during the motivational cue predicted hippocampal activation during encoding of the subsequent scene; across participants, the strength of this interaction predicted memory advantages due to motivation. Of note, punishment-motivated learning was not associated with activation of dopaminergic midbrain, as would be predicted by valence-independent models of motivation to learn. These data are consistent with the view that motivation by punishment activates the amygdala, which in turn prepares the medial temporal lobe for memory formation. The findings further suggest a brain system for declarative learning motivated by punishment that is distinct from that for learning motivated by reward.
\end{abstract}

\section{Introduction}

When faced with a threat, individuals are motivated to seek out and encode information to help avoid it. Candidate neural circuitry for the influence of motivation on declarative memory has been described under conditions of reward, but potential mechanisms under conditions of punishment remain open. In particular, punishment-motivated memory encoding could rely on the same systems guiding reward-motivated encoding or could, alternatively, rely on systems specialized to process environmental threat. The current study characterized neural mechanisms of motivated declarative encoding when individuals expected that forgetting would be punished by mild electrical shocks.

Motivated behaviors, including declarative learning and memory (Shohamy and Adcock, 2010), have been demonstrated to be dependent on mesolimbic dopamine systems, especially the ventral tegmental area (VTA) (Berridge and Robinson, 1998; Wise, 2004).

\footnotetext{
Received Jan. 6, 2012; revised April 25, 2012; accepted May 9, 2012.

Author contributions:V.P.M., K.S.L., and R.A.A. designed research;V.P.M., K.S.L., and R.A.A. performed research; V.P.M. analyzed data; V.P.M., K.S.L., and R.A.A. wrote the paper.

This project was supported by National Institutes of Health Grants R01 DA027802 and 2P01 NS41328-06. We thank M. Ritchey, J. Macinnes, P. Kragel, and S. Prince for helpful discussions and comments. We thank K. Macduffie, R. Loiotile, and A. Susac for assistance with data collection and analysis.

The authors declare no competing financial interests.

Correspondence should be addressed to R. Alison Adcock, B203 Levine Science Research Center, Duke University, Box 90999, Durham, NC 27708. E-mail: alison.adcock@duke.edu.

DOI:10.1523/JNEUROSCI.0094-12.2012

Copyright $\odot 2012$ the authors $\quad 0270-6474 / 12 / 328969-08 \$ 15.00 / 0$
}

During reward-motivated declarative encoding, interactions between the VTA and hippocampus predict successful memorization of incentivized information (Adcock et al., 2006). In humans, reward and punishment motivation have both been associated with activation throughout the mesolimbic dopamine system, including the VTA (Carter et al., 2009; Delgado et al., 2011). Because punishment motivation, like reward motivation, engages the VTA, it is possible that punishment-motivated encoding could also engage the VTA. Alternatively, punishment-motivated encoding may depend on amygdala neuromodulation. The amygdala and its interactions with the medial temporal lobe have been critically implicated in enhancing memory for intrinsically threatening stimuli (LaBar and Cabeza, 2006), while in humans the VTA is not reliably activated during this type of emotional memory encoding (Murty et al., 2010).

The separate literatures on motivation and threat processing thus make distinct predictions about the neural systems supporting punishment-motivated declarative encoding. If punishment incentives evoke equivalent motivational states to reward incentives, the VTA should modulate declarative encoding. However, if punishment incentives lead individuals to perceive motivational cues or memoranda as environmental threats, the amygdala should modulate encoding. Additionally, the amygdala and VTA could facilitate learning jointly (Lalumiere et al., 2004; Darvas et al., 2011).

The goal of this study was to provide evidence to adjudicate these theoretical perspectives regarding the neural circuitry underlying punishment-motivated declarative encoding. During 
functional magnetic resonance imaging (fMRI), motivational cues instructed participants whether forgetting memoranda (target scenes) would or would not be punished with a mild electrical shock (see Fig. 1). Twenty-four hours after encoding, participants were tested for scene recognition but were not in fact punished to isolate the effects of punishment motivation during encoding. Analyses investigated how the threat of shock influenced recognition memory, encoding success activation (ESA; an activation that is stronger for subsequently remembered versus forgotten memoranda), and functional connectivity. Critically, fMRI activation and functional connectivity were analyzed both before and during target scene encoding. Analyses aimed to delineate relative contributions of the VTA and amygdala and their interactions with the medial temporal lobe during punishment-motivated encoding, thus informing models of both active avoidance and motivated memory.

\section{Materials and Methods}

\section{Participants}

Twenty-five healthy, right-handed volunteers participated in this study. All participants gave written informed consent for a protocol approved by the Duke University Institutional Review Board (Durham, NC). We excluded seven participants because of excessive head motion $(>1.5$ $\mathrm{mm}$, two participants), insufficient trial types in conditions of interest (four participants), or software malfunction during scanning (one participant), which resulted in 18 participants (eight female; age range, 1837; median age, 24) for the final analysis.

\section{Task}

FMRI was performed during a shock incentivized encoding task. During this task, participants studied scene images to avoid punishment in the form of electrical stimulations for memory failures at a $24 \mathrm{~h}$ retrieval test. On each trial, participants first viewed a motivational cue indicating the value of encoding an upcoming target scene. Participants either viewed a shock cue (a lightning bolt), which indicated that participants could avoid receiving an electrical shock at a $24 \mathrm{~h}$ recognition test by successfully encoding the upcoming scene, or a no-shock cue (a lightning bolt with an overlaid cancel sign), which indicated that there was no threat of shock associated with memory for the upcoming scene (Fig. 1). Regardless of the cue, we instructed participants to try to encode all stimuli. Following the cue $(1 \mathrm{~s})$, participants viewed a fixation cross $(2.5-6.5 \mathrm{~s})$, followed by the target scene to be encoded $(2 \mathrm{~s})$. Target scenes were color photographs of indoor and outdoor scenes, each of which was novel. Pictures were pseudorandomly divided into four sets of 60 that contained equal proportions of indoor and outdoor scenes. Assignments to study stimuli or recognition test foils were counterbalanced across participants. Following each target scene, participants performed a visual motor distractor task to prevent further elaboration of the target and to allow for an active baseline for neuroimaging analyses (Stark and Squire, 2001). This distractor task required participants to indicate the direction of an arrowhead (left or right; three trials totaling $2.5 \mathrm{~s}$, arrow duration $667 \mathrm{~ms}$, $250 \mathrm{~ms}$ interstimulus interval) with a rapid congruent button press. Following the distractor task, participants viewed a screen with no fixation cross (4.5-18.5 s) and were instructed to remain alert for the next cue. Participants received 60 shock and 60 no-shock trials pseudorandomized over four runs, each lasting $8 \mathrm{~min}$ and $34 \mathrm{~s}$. Trial onsets, cue scene intervals, and trial order were optimized using Optseq software (http://surfer.nmr.mgh.harvard.edu/optseq).

\section{Procedures}

Before scanning, participants calibrated electrical shocks to a level that was "highly irritating but not painful" using an ascending staircase pro- cedure with $5 \mathrm{mV}$ increments (Dunsmoor et al., 2012). Shocks were administered using the MP-150 BIOPAC system (BIOPAC Systems). Following shock calibration, participants were given instructions for the task. Participants were told that they would see a series of scene images preceded by motivational cues and that memory for scenes would be tested at a $24 \mathrm{~h}$ delay memory test. Participants were informed that each trial began with one of two cues, a shock or a no-shock cue. Critically, participants were instructed that they would not receive any shocks during any runs of the task. Participants were instructed that they could avoid receiving shocks at the $24 \mathrm{~h}$ retrieval session by successfully remembering scene images following shock cues, and that there was no threat of shock associated with scenes following no-shock cues.

Inside the scanner, participants first performed four runs of the task. Before each run of the task, participants were given a single demonstration of the shock to increase the efficacy of the motivational cues. Following the task, participants performed one run of the localizer task (described below in the last paragraph of this section). Upon leaving the scanner, participants completed a postscanning questionnaire in which they were asked to report how motivating they found the shock incentives on a scale of 1 (not motivating) to 5 (extremely motivating). Participants were then informed that the threat of shock was an instructional manipulation only and no shock would be given during the $24 \mathrm{~h}$ retrieval session; however, their memory would still be tested.

The following day (22-26 h after the encoding session), participants performed a recognition memory task for the target scenes. During this test, participants saw 120 studied scenes ( 60 shock, 60 no-shock) and 120 novel foil scenes in a randomized order. For each scene image, we asked participants to decide whether each picture was "OLD" or "NEW" by pressing the " 8 " and " 9 " buttons, respectively, on a keyboard. Following each memory decision, participants had to indicate their confidence in their response (i.e., 1 = Very Sure, $2=$ Pretty Sure, $3=$ Just Guessing).

The scanned encoding session also included a functional localizer for defining amygdala regions of interest (ROIs). In this task, participants viewed blocks of trial-unique, fearful male and female faces derived from a standard set of pictures of facial affect (Ekman and Friesen, 1976) or gray oval control stimuli with the letter " $M$ " or " $F$ " (modified from Hall et al., 2008). Participants received three blocks each of fearful faces and control stimuli. During each block participants viewed six stimuli (either all faces or all control stimuli) for $3.5 \mathrm{~s}$ each with an interstimulus interval of $0.8 \mathrm{~s}$ (block length, $25.8 \mathrm{~s}$ ) and responded to indicate the gender or letter.

\section{MRI data acquisition and preprocessing}

FMRI data were acquired on a 3.0T GE Signa MRI scanner using a standard echo-planar sequence (TE, $27 \mathrm{~ms}$; flip, $77^{\circ}$; TR, $1 \mathrm{~s}$; 17 contiguous slices; size, $3.75 * 3.75 * 3.80 \mathrm{~mm}$ ). Partial brain acquisition with a short TR was used to maximize the sampling rate in regions of interest. Brain images were acquired in an orientation that maximized coverage of the medial temporal lobe, striatum, and medial prefrontal cortex, and thus excluded motor cortex, parietal cortex, and parts of the dorsal visual stream. Task data were acquired in four runs with 514 volumes each $(8$ min and $34 \mathrm{~s}$ ). The first 12 volumes of each functional run were removed to allow magnetic stabilization. Before the functional runs, a whole-brain 
inversion recovery spoiled gradient recalled (IR-SPGR) high-resolution anatomical image (voxel size, $1 \mathrm{~mm}$, isotropic) was collected for spatial normalization. Before each run, a whole-brain echo-planar imaging (EPI; 34 contiguous slices) was collected with the same voxel size and orientation as partial EPI brain images to assist with normalization.

FMRI preprocessing was performed using SPM8 (www.fil.ion. ucl.ac.uk/spm) software to first realign across runs and then smooth the data using an isotropic $4 \mathrm{~mm}^{3}$ FWHM (full width at half maximum) kernel. Data were visually inspected on custom software to review for head motion and artifacts. Data were analyzed only if they exhibited $<1.5$ $\mathrm{mm}$ motion (absolute maximum). Images with transient noise artifacts $(>2.5 \mathrm{SD}$ from mean) were replaced with interpolated data from neighboring time points (artifacts occurred in $<1.5 \%$ of the data). Preprocessed functional images (partial brain EPI) were first registered to whole-brain EPI images. Then whole-brain EPI images were normalized to the MNI template using a fifth degree B-spline interpolation. The normalization matrix was applied to the realigned functional images.

\section{Behavioral analysis}

Self-reports of motivation to learn were submitted to a one-sample $t$ test. To measure differences in memory across conditions, correctedrecognition scores (Hits - False Alarms) were calculated separately for scenes following shock cues and no-shock cues, excluding guesses. Corrected recognition scores were calculated separately for all responses and responses endorsed with confidence ("pretty sure" and "very sure" responses). Corrected-recognition scores were then compared across shock and no-shock conditions using a paired $t$ test. Statistical thresholds were set at $p<0.05$.

\section{fMRI data analysis}

General linear model. MRI data analysis was performed using SPM8. We modeled individual subject fMRI data using a general linear model (GLM). Separate regressors were created modeling the cue and target scene onsets, with event durations specified as 0.5 and $1 \mathrm{~s}$, respectively. In both cases, task regressors were convolved with a canonical hemodynamic response function before analysis. Imaging data were normalized to the mean global signal across the functional volumes throughout the entire session and high-pass filtered $(<124 \mathrm{~s})$.

A parametric design was used to investigate the effects of motivation condition and encoding success. Condition regressors weighted all trials equally for each of the (cue/target) $\times$ (shock/no-shock) event types. To investigate parametric modulation of brain activity by encoding success, additional regressors were constructed for each subject for each event type weighted according to the subject's memory strength at retrieval for the scene presented on that trial (parametric ESA). Thus, trial weights for these regressors were greatest when scenes were most strongly remembered, intermediate when they were weakly remembered, and lowest when they were forgotten. Specifically, for each participant we calculated the corrected recognition score (hits-false alarms) for stimuli endorsed as old with each confidence rating (Very Sure, Pretty Sure, and Just Guessing) at retrieval, with a value of zero assigned to trials in which scenes were subsequently forgotten. Thus, for each individual participant the values for the shock parametric ESA would be calculated as follows: Shock Hit (VerySure) - False Alarm (VerySure) , Shock Hit ${ }_{(\text {PrettySure) }}-$ False Alarm $_{\text {(PrettySure), Shock Hit }}$ (Just Guessing) $_{\text {- False Alarm }}$ (Just Guessing), Miss $=0$. For example, for a single individual these parameters might be: Very Sure $(0.45-0.10=0.35)$; Pretty Sure $(0.35-0.20=0.15)$; Just Guessing $(0.02-0.01=0.01)$; Forgotten $(0)$. Individualized correctedrecognition scores for each confidence rating were then assigned to all the trials that elicited that confidence rating. Similar parametric analyses have previously been used to investigate encoding success activations in functional imaging data (Kensinger et al., 2011; Ritchey et al., 2011). Using the GLM, individual maps of parameter estimates were generated for contrasts of interest: shock cue $>$ no-shock cue, shock target scene $>$ no-shock target scene, parametric ESA shock cue $>$ no-shock cue, and parametric ESA shock target scene $>$ no-shock target scene. Group-level random effects analyses were performed using one-sample $t$ tests.

ROI analyses. ROI analyses were performed to confirm and visualize findings from the whole-volume GLM, directly compare the contribu- tions of VTA and amygdala, and independently investigate brainbehavior relationships across individuals. ROIs were defined as follows: mean $\beta$-parameters were extracted from contrasts of interest from $8 \mathrm{~mm}$ spheres centered around peak coordinates derived from the amygdala localizer task and $8 \mathrm{~mm}$ spheres around peak coordinates in the VTA derived from a prior study investigating rewardmotivated declarative memory encoding (Adcock et al., 2006). Amygdala ROIs were defined separately for each participant by intersecting peak activations from a contrast of fearful $>$ control blocks in the independent localizer task (as determined by $t$ statistic) with left and right amygdala anatomical ROIS defined using the WFU PICKATLAS (http://www.fmri.wfubmc.edu/cms/software).

To confirm whole-volume GLM analyses in the VTA and amygdala, we submitted extracted $\beta$-parameters to one-sample $t$ tests. To directly compare the contributions of VTA and amygdala, we submitted $\beta$-parameters extracted from the left and right VTA and amygdala, respectively, to a twofactor, within-subject GLM. To investigate brain-behavior relationships across individuals, we regressed individual extracted $\beta$-parameters against corrected recognition scores for scenes following shock versus no-shock cues, with guessed responses omitted.

Within-subject amygdala connectivity/interactions. To assess functional interactions between the right amygdala and other neural regions, withinsubjects regression analyses were performed using single-trial $/ \beta$-series analysis (for a similar approach see Rissman et al., 2004; Ritchey et al., 2008). Connectivity analyses were limited to the right amygdala because GLM analyses were only significant on the right. A GLM was constructed for each participant that separately modeled cue and scene-target activations for each individual trial. Then, cue-related $\beta$-values were extracted for each trial from the right amygdala ROI independently defined by the functional localizer. Next, for each participant, GLM/multiple regressions of single-trial data were constructed to identify neural regions that were correlated with the amygdala $\beta$-series as a function of motivational cue ( shock $>$ cue) or as a function of motivational cue and parametric encoding success interactions. Finally, separate GLMs were constructed to investigate how amygdala cue-related activations interacted with cuerelated activations across all other voxels and target scene-related activations across all other voxels. The former analysis identified regions that were functionally connected with the amygdala during motivational cue presentations on a trial-by-trial basis, while the latter analysis identified regions where preparatory activations in the amygdala predicted responses to subsequent target-scene images on a trial-by-trial basis. Then, we generated individual maps of parameter estimates reflecting amygdala interactions for contrasts of interest and entered these into group level, random effects, one-sample $t$ tests.

Statistical thresholds. Statistical tests for whole-volume neuroimaging analyses were thresholded at significance of $p<0.001$ for spatial extent for multiple comparisons correction yielding a cluster extent minimum of 14 voxels. This procedure corresponds to an overall $\alpha=0.05$ familywise error rate, as calculated within AlphaSim (http://afni.nimh.nih.gov/ afni/doc/manual) with 1000 Monte Carlo simulations.

\section{Results}

\section{Behavioral results}

Analysis of self-reports revealed that the threat incentive significantly increased participants' motivation to perform on shock trials $\left(t_{(17)}=18.327, p<0.001\right)$. Twenty-four hour delayed memory recognition for target scenes that followed shock cues $\left(t_{(17)}=9.821, p<0.001\right)$ and no-shock cues $\left(t_{(17)}=7.189, p<\right.$ $0.001)$ was significantly greater than false alarms to new scenes (mean \pm SEM: shock $=53.7 \pm 2.2 \%$, no-shock: $47.7 \pm 3.02 \%$, false alarm: $27.4 \pm 2.60 \%)$. The threat of shock enhanced scene encoding, such that corrected recognition rates (hits - false alarms, excluding guessing responses) were greater for target scenes following shock cues compared to no-shock cues $\left(t_{(17)}=\right.$ $4.281, p=0.001$, Fig. 2). This comparison remained significant when all responses were included $\left(t_{(17)}=2.957, p=0.009\right)$. 


\section{fMRI results}

Main effect of shock

To identify brain regions that were modulated by the threat of shock independent of memory, we compared activations across shock and no-shock trials. During the presentation of motivational cues, the contrast of shock cues compared to no-shock cues revealed greater activation in the right anterior cingulate cortex and less activation bilaterally in the fusiform gyrus (Table 1). During the presentation of target scenes, the threat of shock resulted in greater activation compared to no-shock in the right caudate/ventral striatum, left putamen, and bilateral fusiform gyrus, and less activation in the right superior frontal gyrus (Table 1).

\section{Main effect of encoding success}

To identify which brain regions were more activated during successful than unsuccessful encoding (i.e. ESA), we used regressors parametrically related to memory strength at later recognition, regardless of trial type. There were no ESAs during the presentation of cues. However, during the presentation of target scenes, posterior parahippocampal cortex showed significant ESAs, indicating that activation in this region increased with memory encoding strength (Table 1).

\section{Threat of shock and encoding success interactions}

To isolate regions implicated in memory encoding selectively during motivation to avoid punishments, we compared ESA parameter estimates in shock versus no-shock trials. We hereafter refer to these contrasts as "shock-motivated ESAs." During the presentation of cues, shock-motivated ESAs were seen only in the right amygdala (Fig. 3A). No significant interactions were seen in the reverse contrast. During the presentation of target scenes, neither shockmotivated ESAs nor the reverse contrast was significant.

The complimentary ROI analyses also demonstrated shock-motivated ESAs in the right, but not left, amygdala (left: $t_{(17)}=1.705, p=0.11$; right: $t_{(17)}=$ 2.553, $p=0.02$; Fig. $3 B$ ). Interestingly, individual differences analysis revealed that amygdala activation during the presentation of cues predicted learning under threat; across subjects, shockmotivated ESAs from the right amygdala were positively correlated with shockmotivated recognition memory advantages $\left(F_{(17)}=6.858, p=0.03, r=0.51\right.$; Figure $3 C)$. During the presentation of target scenes, there were not any shockmotivated ESAs in the amygdala ROIs (left: $t_{(17)}=0.38, p=0.71$; right: $t_{(17)}=$ $0.72, p=0.48)$.

Regarding the dopaminergic midbrain, whole-volume analysis revealed no significant activations in the VTA or the substantia nigra, even at a lenient threshold of $p<0.05$ uncorrected. Furthermore, ROI analyses of the VTA revealed that there were not any shock-motivated ESAs during the presentation of the cues (left: $t_{(17)}=-0.004, p=0.997$, right: $t_{(17)}=-0.75, p=0.464$; Fig. $3 B$ ) or target scenes (left: $t_{(17)}=1.14$, $p=0.27$; right: $t_{(17)}=1.59, p=0.13$ ).

To explicitly test whether punishment-motivated encoding was more associated with the amygdala compared to the VTA, $\beta$-parameters from shock-motivated ESAs at the presentation of the cue were submitted to a two-factor (laterality and region), withinsubjects GLM. This analysis revealed a main effect of region $\left(F_{(17)}=\right.$

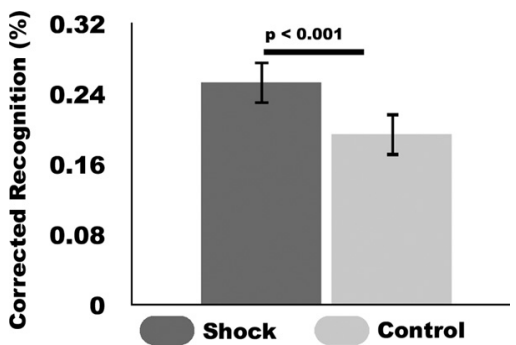

Figure 2. Threat of shock influences scene encoding. Mean corrected recognition scores (Hits - False Alarms) for scenes following shock and no-shock cues (errors bars represent \pm SEM). Depicted results exclude trials in which memory recognition was endorsed with "Just Guessing" confidence responses.

\section{Table 1. Significant activations from threat of shock manipulation}

\begin{tabular}{lrrrrr}
\hline Region & \multicolumn{1}{c}{$x$} & $y$ & $z$ & $Z$ & $k$ \\
\hline Cue: shock $>$ no-shock & & & & & \\
$\quad$ Anterior cingulate & 18 & 32 & 12 & 3.6 & 64 \\
$\quad \begin{array}{l}\text { Cue: } \text { no-shock }>\text { shock } \\
\quad \text { Fusiform gyrus }\end{array}$ & -28 & -56 & -12 & 3.98 & 114 \\
$\quad$ Fusiform gyrus & 32 & -60 & -14 & 3.45 & 28 \\
$\quad \begin{array}{l}\text { Target scene: shock > no-shock } \\
\quad \text { Caudate/ventral striatum }\end{array}$ & -6 & 6 & 2 & 4.38 & 83 \\
$\quad$ Fusiform gyrus & -48 & -42 & -20 & 3.65 & 44 \\
$\quad$ Fusiform gyrus & -46 & -58 & -14 & 3.59 & 20 \\
$\quad$ Putamen & 18 & 12 & -4 & 3.34 & 24 \\
$\quad$ Target scene: no-shock > shock & & & & & \\
$\quad$ Superior frontal gyrus & -26 & 58 & 20 & 3.73 & 18 \\
$\quad$ Cue: encoding success activations & & & & & \\
$\quad$ No significant activations & & & & & \\
$\quad$ Target: encoding success activations & & & & & \\
$\quad$ Parahippocampus/fusiform gyrus & 32 & -48 & -16 & 4.12 & 108 \\
\hline
\end{tabular}

$x, y, z=$ MNI coordinates; $k=$ cluster size; $Z=z$-score.

Figure 3. Threat of shock increases preparatory encoding success activations, ESAs, in the amygdala. $\boldsymbol{A}$, The right amygdala showed greater encoding success activations in response to shock cues compared to no-shock cues. $\boldsymbol{B}$, Beta-parameters extracted from a-priori ROIs confirmed significant shock-motivated ESA (shock cue ESA $>$ no-shock cue ESA) in the right amygdala, but not the left amygdala or bilaterally in the VTA. C, Across participants, shock-motivated ESAs during cue presentations were positively correlated with the extent to which memory was enhanced under threat of shock.

$8.446, p=0.01)$, such that shock-motivated ESAs were greater in the amygdala compared to the VTA. Neither the laterality nor interaction term was statistically significant $(F<1)$.

\section{Amygdala functional connectivity}

To investigate how functional connectivity between the amygdala and other regions contributed to learning under threat, we ran a within-subjects, single-trial analysis on cue-related activations with the right amygdala functional ROI as the seed. During the presentation of cues, we found a significant interaction between condition (shock $>$ no-shock cues) and encoding success connectivity, such that amygdala connectivity with the right parahip- 

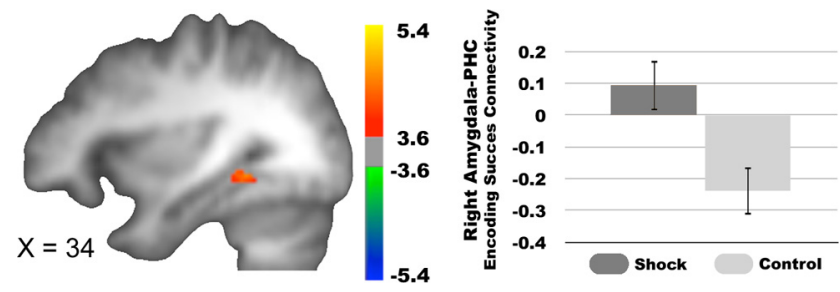

Figure 4. Amygdala-parahippocampus connectivity and encoding success. The right amygdala and parahippocampus (PHC) showed significantly greater encoding success-related functional connectivity on a trial-by trial basis, which is greater for strongly remembered $>$ forgotten stimuli, following shock cues compared to no-shock cues.

A

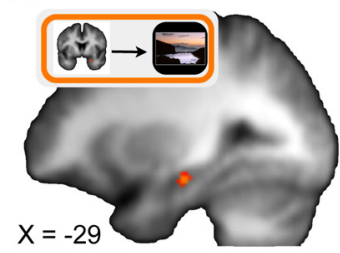

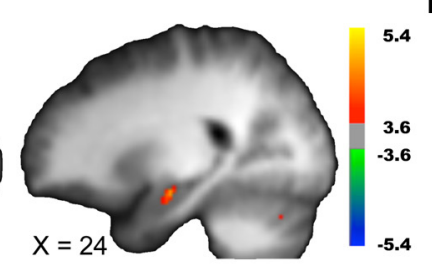

B

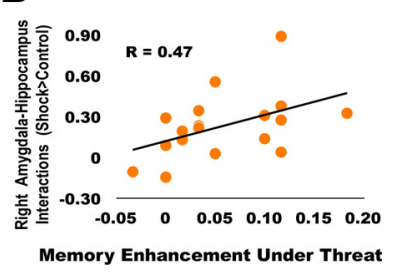

Figure 5. Influence of preparatory amygdala activation on hippocampal scene encoding. $A$, Cue-related activation in the right amygdala showed greater covariation with hippocampal scene encoding bilaterally during shock trials compared to no-shock trials. $\boldsymbol{B}$, The extent of this covariation in the right hippocampus was positively correlated with the extent to which an individual's memory was enhanced under the threat of shock.

Table 2. Significant interactions between the cue-related activations in the right amygdala and scene encoding activations

\begin{tabular}{lrrrrr}
\hline Amygdala interactions with target processing & $x$ & $y$ & $z$ & $Z$ & $k$ \\
\hline Shock $>$ No-shock region & & & & & \\
$\quad$ Cerebellum & 44 & -58 & -40 & 3.74 & 38 \\
& 4 & -38 & -12 & 3.68 & 103 \\
& 10 & -44 & -44 & 3.60 & 93 \\
& 10 & 52 & -16 & 3.27 & 17 \\
Hippocampus & -8 & -54 & -12 & 3.42 & 21 \\
& 26 & -12 & -16 & 3.66 & 37 \\
Middle temporal gyrus & -26 & -20 & -14 & 3.47 & 24 \\
Insula & 48 & -14 & -12 & 3.63 & 23 \\
No-shock $>$ shock & -32 & 22 & -6 & 3.36 & 14 \\
No significant interactions & & & & & \\
\hline$x, y, z=$ MNI coordinates; $k=$ cluster size; $z=z$-score. & & & & & \\
\end{tabular}

pocampal cortex $(x, y, z=34,-44,-6$; cluster extent $=23 ; Z=$ $3.54)$ and the right orbitofrontal cortex $(x, y, z=40,40,-10$; cluster extent $=15 ; Z=3.78$ ) predicted encoding success to a greater extent following shock than no-shock cues (Fig. 4).

Amygdala cue and target-scene interactions

To investigate whether motivational cue-evoked activity in the amygdala modulated processing of the upcoming target scene, we ran a single-trial analysis between cue-evoked activations in the amygdala and target scene-evoked activations on the same trial relative to no-shock. The threat of shock increased trial-by-trial covariation between cue-evoked activations in the right amygdala and scene-evoked activations bilaterally in the hippocampus (Fig. 5A) as well as in the bilateral cerebellum, right middle temporal gyrus, and left insula (Table 2). Across participants, right amygdala-right hippocampus covariation predicted shock-motivated recognition memory advantages $\left(F_{(17)}=4.449\right.$, $p=0.051, r=0.47$; Fig. 5B).

\section{Discussion}

Although previous research has examined the effects of motivation on declarative memory encoding, this literature is limited and has focused on motivation to gain rewards. Here we examined how an alternative motivator, the threat of punishment, influenced declarative memory encoding. We found that motivating individuals to memorize images with the threat of mild electrical shocks for forgetting resulted in better memory for those images. Successful declarative encoding under the threat of shock was associated with amygdala but not VTA engagement. Specifically, threat-related encoding was associated with right amygdala activation and functional connectivity of right amygdala with parahippocampal and orbitofrontal cortex. Interestingly, engagement of these functional networks preceded successful encoding, suggesting a preparatory role for amygdala neuromodulation. Finally, we found that preparatory activations in the right amygdala predicted scene-evoked activations in the hippocampus and further predicted punishment-motivated encoding performance across participants. Together, our findings demonstrate that the threat of shock engages amygdala rather than dopaminergic midbrain prior to motivated encoding. Furthermore, they suggest that neuromodulatory signals from the amygdala influence different regions first during a threat cue and then during encoding of the memorandum to support punishment-motivated memory.

\section{Punishment motivation enhances scene learning}

The current findings demonstrate that punishment motivation enhances intentional encoding, in that individuals better recognized scenes under the threat of punishment. Although previous studies have described better declarative memory for intrinsically threatening stimuli (Hamann, 2001; LaBar and Cabeza, 2006; Davis et al., 2011) and neutral stimuli paired with threats (Dunsmoor et al., 2012; Schwarze et al., 2012), the efficacy of punishment motivation on neutral stimuli has not been investigated. Our findings show that avoidance motivation, like other forms of motivation (Adcock et al, 2006; Callan and Schweighofer, 2008; Kang et al., 2009; Murayama and Kuhbandner, 2011), can enhance declarative memory. However, declarative memory facilitation by punishment motivation observed in the current study may be limited to certain types of learning, such as list learning. During spatial navigation, punishment motivation has been shown to result in memory deficits (Murty et al., 2011). Thus, learning under threat may enhance some, but not all, forms of declarative memory.

We also demonstrated that threat of shock, even in the absence of actual punishment, enhances declarative learning. Here, motivation was induced purely by experimenter instruction, as shocks were never administered. Although previous animal studies have investigated the role of negative reinforcement on memory encoding (McGaugh, 2004), these studies were not able to dissociate motivation from the actual receipt of aversive stimuli. Thus, we have provided a novel characterization of how avoidance motivation influences declarative learning, even in the absence of punishment delivery. 


\section{Amygdala activation predicts punishment-motivated memory encoding}

Our fMRI analyses revealed that, under the threat of shock, right amygdala activation following shock compared to noshock cues predicted punishment-motivated encoding, both during whole-volume and ROI analyses. Individual differences analyses revealed that these preparatory activations in the amygdala predicted punishment-motivated memory enhancements across participants. These findings suggest that neuromodulatory signals from the amygdala prepare individuals to encode information to avoid future punishments.

Previous research has implicated the amygdala in human avoidance behavior (Lang and Bradley, 2009), which we here extend to include intentional declarative learning. Neuroimaging studies have revealed amygdala activation when individuals prepare motor responses to avoid penalties and punishments (Mobbs et al., 2007, 2009; Schlund and Cataldo, 2010; Delgado et al., 2011) and learn simple stimulus-response contingencies to avoid penalties (Prévost et al., 2011). Thus, like most rodent studies, human active avoidance procedures typically investigated the implementation of simple motor behaviors to avoid punishment. Our study extends the human active avoidance literature by demonstrating that the amygdala's role in avoidance behaviors can also encompass more cognitive processes, such as instrumental declarative memory encoding.

Our findings also expand the amygdala's role in declarative memory to include motivational effects on neutral material. A recent meta-analysis identified the right amygdala as the most consistently activated region during the successful encoding of intrinsically emotional stimuli (Murty et al., 2010). Furthermore, during the anticipation of aversive events, preparatory signals from the amygdala contribute to emotional memory encoding (Mackiewicz et al., 2006). The current study shows that the amygdala's involvement in declarative memory is not limited to intrinsically threatening stimuli, but also includes neutral stimuli associated with potential future punishment.

\section{Functional interactions with the amygdala predict punishment-motivated memory encoding}

Results revealed that functional connectivity of the right amygdala with the parahippocampal and orbitofrontal cortices predicted punishment-motivated scene encoding. Specifically, under threat of shock, positive coupling of the right amygdala with the right parahippocampal and orbitofrontal cortices before scene encoding predicted encoding success. The amygdala and orbitofrontal cortex are strongly interconnected in nonhuman primates (Porrino et al., 1981; Amaral and Price, 1984; Ghashghaei and Barbas, 2002), and interactions between these regions have been demonstrated to support the valuation of aversive events in rodents (Schoenbaum et al., 2007) and nonhuman primates (Salzman et al., 2007). The current study only found significant differences in amygdala-orbitofrontal cortex connectivity and did not reveal any significant differences in orbitofrontal activations (even at a lenient statistical threshold of $p<$ 0.01 uncorrected). This pattern suggests that the role of the orbitofrontal cortex in punishment-motivated encoding may only emerge via neuromodulatory interactions with the amygdala. The amygdala is also strongly interconnected with the parahippocampal cortex in nonhuman primates (Suzuki and Amaral, 1994), and models of declarative memory have identified the parahippocampal cortex to be critical for transferring contextual information to the hippocampus (Davachi, 2006; Ranganath, 2010). Furthermore, amygdala-parahippocampal interactions have previously been associated with declarative memory encoding for emotionally arousing stim- uli and contexts (Dolcos et al., 2004; Alvarez et al., 2008; Ritchey et al., 2008; Rudy, 2009). Thus, previous research provides the anatomical and functional foundation whereby amygdala interactions with the orbitofrontal and parahippocampal cortices could support punishment-motivated scene encoding.

The current results also revealed significant functional interactions between the amygdala and hippocampus during punishmentmotivated encoding. However, the temporal dynamics of these interactions differed from amygdala interactions with parahippocampal and orbitofrontal cortices. Specifically, under threat of shock, preparatory, cue-evoked amygdala activations predicted subsequent scene-evoked hippocampus activations. This pattern of relationships suggests that motivational signals arising from the amygdala first modulate parahippocampal cortex before encoding and then subsequently modulate hippocampus during encoding.

Beyond temporal dynamics, the relationships with performance were different for amygdala connectivity with hippocampus versus parahippocampal cortex. Whereas amygdala interactions with parahippocampal cortex predicted punishment-motivated memory enhancements on a trial-by-trial basis, interactions with the hippocampus predicted enhancements only across participants. This difference in temporal dynamics and brain-behavior relationships suggests that the hippocampus and parahippocampal cortex have different mechanisms for facilitating punishment-motivated encoding. Modulation of parahippocampal cortex could reflect enhancements of perceptual processing of scenes, given its position in the ventral visual stream, and could thus account for trial-by-trial variations in memory encoding strength. However, the relationship between punishment-motivated encoding and amygdalahippocampus interactions was across subjects. This relationship suggests that mechanisms acting on a slower timescale were facilitating memory encoding by the hippocampus. One potential mechanism could be the triggering of noradrenaline- and glucocorticoid-mediated memory consolidation, which has been associated with phasic activation of limbic circuitry but a delayed memory enhancement that may not initiate until 20 min postencoding (Joëls et al., 2011).

In light of our findings, we propose a model of punishmentmotivated declarative learning in which the amygdala transfers valuation signals from the orbitofrontal cortex to prepare both the parahippocampal cortex and hippocampus for future encoding. Consistent with this interpretation, a recent neuroimaging study showed that the amygdala mediates the relationship between orbitofrontal cortex and medial temporal lobe when individuals update aversive memories (Sakaki et al., 2011). We suggest that the orbitofrontal cortex-amygdala interaction observed in the current study facilitates two distinct mechanisms in the medial temporal lobes to promote declarative learning: a more perceptually driven facilitation of parahippocampal cortex that precedes learning perhaps as a priming mechanism, and a state-dependent facilitation of hippocampus that may reflect consolidation mechanisms.

\section{Implications for motivated memory}

Our findings provide a novel characterization of the neural mechanisms mediating punishment-motivated declarative encoding and have important implications for models of motivated memory. Critically, our results demonstrate that punishmentmotivated encoding is associated with amygdala neuromodulation rather than the dopaminergic midbrain. Analyses did not reveal any role for the VTA during punishment-motivated encoding, even at liberal thresholds. Using a similar design, our 
laboratory has found that reward cues engage preparatory activations in and coupling between the VTA and hippocampus to facilitate reward-motivated memory encoding (Adcock et al., 2006). Together, these findings demonstrate that reward and punishment motivation can engage unique neuromodulatory systems to support medial temporal lobe-dependent encoding. When these systems are selectively engaged, reward and punishment may differentially influence memory representations. In line with this interpretation, reward and punishment motivation can have opposing effects during spatial learning in a modified virtual Morris water maze task (Murty et al., 2011). Future studies are warranted to determine the relative effectiveness of these motivators across a variety of learning experiences, including those in educational contexts.

The current study provides initial evidence for distinct systems guiding punishment-motivated compared to rewardmotivated encoding. Here, the signal averaging requirements and overall session length needed to conduct subsequent memory analyses precluded incorporating a reward motivation manipulation into the single session; thus, future studies will be needed to directly compare activations across these two motivational states. However, these future studies would need to address context effects of the incentives on each other, as well as on overall encoding. The shock cues used here may have evoked context-dependent modulations in encoding, as has been demonstrated during the encoding of intrinsically emotional stimuli (Anderson et al., 2006; Henckens et al., 2009; Knight and Mather, 2009); indeed, overall memory performance was lower for both conditions than in our prior report using a rewardmotivation manipulation (Adcock et al., 2006). Future studies will be needed to investigate whether punishment motivation results in a general modulation of memory for all scenes. If so, these studies will need to characterize the neural mechanisms of such context-dependent modulation.

\section{Conclusions}

The present study investigated neural systems underlying declarative memory encoding when individuals were threatened with punishment for forgetting. Successful encoding under threat was associated with activation of the amygdala, not the VTA, supporting valence-specific models of motivation to learn. Amygdala activation was evoked by mere threat and predicted memory advantages even in the absence of punishment, implying that threat expectations themselves can shape the contents of memory. We found that memory was enhanced when the amygdala interacted with the parahippocampal and orbitofrontal cortices before and with the hippocampus during experiences that were later remembered. The disparate time courses of cortical and hippocampal interactions with the amygdala suggest synergistic mechanisms to enhance memory in service of avoiding future punishments.

\section{References}

Adcock RA, Thangavel A, Whitfield-Gabrieli S, Knutson B, Gabrieli JD (2006) Reward-motivated learning: mesolimbic activation precedes memory formation. Neuron 50:507-517.

Alvarez RP, Biggs A, Chen G, Pine DS, Grillon C (2008) Contextual fear conditioning in humans: cortical-hippocampal and amygdala contributions. J Neurosci 28:6211-6219.

Amaral DG, Price JL (1984) Amygdalo-cortical projections in the monkey (Macaca fascicularis). J Comp Neurol 230:465-496.

Anderson AK, Wais PE, Gabrieli JD (2006) Emotion enhances remembrance of neutral events past. Proc Natl Acad Sci U S A 103:1599-1604.

Berridge KC, Robinson TE (1998) What is the role of dopamine in reward: hedonic impact, reward learning, or incentive salience? Brain Res Brain Res Rev 28:309-369.
Callan DE, Schweighofer N (2008) Positive and negative modulation of word learning by reward anticipation. Hum Brain Mapp 29:237-249.

Carter RM, Macinnes JJ, Huettel SA, Adcock RA (2009) Activation in the VTA and nucleus accumbens increases in anticipation of both gains and losses. Front Behav Neurosci 3:21.

Darvas M, Fadok JP, Palmiter RD (2011) Requirement of dopamine signaling in the amygdala and striatum for learning and maintenance of a conditioned avoidance response. Learn Mem 18:136-143.

Davachi L (2006) Item, context and relational episodic encoding in humans. Curr Opin Neurobiol 16:693-700.

Davis FC, Somerville LH, Ruberry EJ, Berry AB, Shin LM, Whalen PJ (2011) A tale of two negatives: differential memory modulation by threat-related facial expressions. Emotion 11:647-655.

Delgado MR, Jou RL, Phelps EA (2011) Neural systems underlying aversive conditioning in humans with primary and secondary reinforcers. Front Neurosci 5:71.

Dolcos F, LaBar KS, Cabeza R (2004) Interaction between the amygdala and the medial temporal lobe memory system predicts better memory for emotional events. Neuron 42:855-863.

Dunsmoor JE, Martin A, LaBar KS (2012) Role of conceptual knowledge in learning and retention of conditioned fear. Biol Psychol 89:300-305.

Ekman P, Friesen VF (1976) Measuring facial movement. Environ Psychol Nonverbal Behav 1:56-75.

Ghashghaei HT, Barbas H (2002) Pathways for emotion: interactions of prefrontal and anterior temporal pathways in the amygdala of the rhesus monkey. Neuroscience 115:1261-1279.

Hall J, Whalley HC, McKirdy JW, Romaniuk L, McGonigle D, McIntosh AM, Baig BJ, Gountouna VE, Job DE, Donaldson DI, Sprengelmeyer R, Young AW, Johnstone EC, Lawrie SM (2008) Overactivation of fear systems to neutral faces in schizophrenia. Biol Psychiatry 64:70-73.

Hamann S (2001) Cognitive and neural mechanisms of emotional memory. Trends Cogn Sci 5:394-400.

Henckens MJ, Hermans EJ, Pu Z, Joëls M, Fernández G (2009) Stressed memories: how acute stress affects memory formation in humans. J Neurosci 29:10111-10119.

Joëls M, Fernandez G, Roozendaal B (2011) Stress and emotional memory: a matter of timing. Trends Cogn Sci 15:280-288.

Kang MJ, Hsu M, Krajbich IM, Loewenstein G, McClure SM, Wang JT, Camerer CF (2009) The wick in the candle of learning: epistemic curiosity activates reward circuitry and enhances memory. Psychol Sci 20:963-973.

Kensinger EA, Addis DR, Atapattu RK (2011) Amygdala activity at encoding corresponds with memory vividness and with memory for select episodic details. Neuropsychologia 49:663-673.

Knight M, Mather M (2009) Reconciling findings of emotion-induced memory enhancement and impairment of preceding items. Emotion 9:763-781.

LaBar KS, Cabeza R (2006) Cognitive neuroscience of emotional memory. Nat Rev Neurosci 7:54-64.

Lalumiere RT, Nguyen LT, McGaugh JL (2004) Post-training intrabasolateral amygdala infusions of dopamine modulate consolidation of inhibitory avoidance memory: involvement of noradrenergic and cholinergic systems. Eur J Neurosci 20:2804-2810.

Lang PJ, Bradley MM (2009) Emotion and the motivational brain. Biol Psychol 84:437-450.

Mackiewicz KL, Sarinopoulos I, Cleven KL, Nitschke JB (2006) The effect of anticipation and the specificity of sex differences for amygdala and hippocampus function in emotional memory. Proc Natl Acad Sci U S A 103:14200-14205.

McGaugh JL (2004) The amygdala modulates the consolidation of memories of emotionally arousing experiences. Annu Rev Neurosci 27:1-28.

Mobbs D, Petrovic P, Marchant JL, Hassabis D, Weiskopf N, Seymour B, Dolan RJ, Frith CD (2007) When fear is near: threat imminence elicits prefrontal-periaqueductal gray shifts in humans. Science 317:1079-1083.

Mobbs D, Marchant JL, Hassabis D, Seymour B, Tan G, Gray M, Petrovic P, Dolan RJ, Frith CD (2009) From threat to fear: the neural organization of defensive fear systems in humans. J Neurosci 29:12236-12243.

Murayama K, Kuhbandner C (2011) Money enhances memory consolidation-but only for boring material. Cognition 119:120-124.

Murty VP, Ritchey M, Adcock RA, LaBar KS (2010) fMRI studies of successful emotional memory encoding: A quantitative meta-analysis. Neuropsychologia 48:3459-3469.

Murty VP, LaBar KS, Hamilton DA, Adcock RA (2011) Is all motivation 
good for learning? Dissociable influences of approach and avoidance motivation in declarative memory. Learn Mem 18:712-717.

Porrino LJ, Crane AM, Goldman-Rakic PS (1981) Direct and indirect pathways from the amygdala to the frontal lobe in rhesus monkeys. J Comp Neurol 198:121-136.

Prévost C, McCabe JA, Jessup RK, Bossaerts P, O’Doherty JP (2011) Differentiable contributions of human amygdalar subregions in the computations underlying reward and avoidance learning. Eur J Neurosci 34: $134-145$.

Ranganath C (2010) A unified framework for the functional organization of the medial temporal lobes and the phenomenology of episodic memory. Hippocampus 20:1263-1290.

Rissman J, Gazzaley A, D’Esposito M (2004) Measuring functional connectivity during distinct stages of a cognitive task. Neuroimage 23:752-763.

Ritchey M, Dolcos F, Cabeza R (2008) Role of amygdala connectivity in the persistence of emotional memories over time: an event-related FMRI investigation. Cereb Cortex 18:2494-2504.

Ritchey M, LaBar KS, Cabeza R (2011) Level of processing modulates the neural correlates of emotional memory formation. J Cogn Neurosci 23:757-771.

Rudy JW (2009) Context representations, context functions, and the parahippocampal-hippocampal system. Learn Mem 16:573-585.

Sakaki M, Niki K, Mather M (2011) Updating existing emotional memories involves the frontopolar/orbito-frontal cortex in ways that acquiring new emotional memories does not. J Cogn Neurosci 23:3498-3514.

Salzman CD, Paton JJ, Belova MA, Morrison SE (2007) Flexible neural representations of value in the primate brain. Ann N Y Acad Sci 1121: $336-354$.

Schlund MW, Cataldo MF (2010) Amygdala involvement in human avoidance, escape and approach behavior. Neuroimage 53:769-776.

Schoenbaum G, Saddoris MP, Stalnaker TA (2007) Reconciling the roles of orbitofrontal cortex in reversal learning and the encoding of outcome expectancies. Ann N Y Acad Sci 1121:320-335.

Schwarze U, Bingel U, Sommer T (2012) Event-related nociceptive arousal enhances memory consolidation for neutral scenes. J Neurosci 32: 1481-1487.

Shohamy D, Adcock RA (2010) Dopamine and adaptive memory. Trends Cogn Sci 14:464-472.

Stark CE, Squire LR (2001) When zero is not zero: the problem of ambiguous baseline conditions in fMRI. Proc Natl Acad Sci U S A 98:1276012766.

Suzuki WA, Amaral DG (1994) Perirhinal and parahippocampal cortices of the macaque monkey: cortical afferents. J Comp Neurol 350:497-533.

Wise RA (2004) Dopamine, learning and motivation. Nat Rev Neurosci 5:483-494 